\title{
Travel to Asia is a strong predictor for carriage of cephalosporin resistant $E$. coli and Klebsiella spp. but does not explain everything; prevalence study at a Norwegian hospital 2014-2016
}

Laura Espenhain ${ }^{1,2^{*}}$ (D) Silje Bakken Jørgensen ${ }^{3}$, Truls Michael Leegaard ${ }^{3,4}$, Michaela Marie Lelek ${ }^{3}$, Siri Haug Hänsgen ${ }^{3}$, Britt Nakstad ${ }^{4,5}$, Marianne Sunde ${ }^{6,7}$ and Martin Steinbakk ${ }^{1}$

\begin{abstract}
Background: We aimed to estimate the prevalence of faecal carriage of extended-spectrum cephalosporin (ESC) resistant E. coli and K. pneumoniae (ESCr-EK) and vancomycin resistant enterococci (VRE) in patients upon hospital admission and identify factors associated with carriage to better target interventions and to guide empirical antibiotic treatment.

Methods: Between October 2014 and December 2016, we recruited patients admitted to a Norwegian university hospital. A rectal swab and questionnaire covering possible risk factors for colonisation were collected upon admission. Isolates were characterized by phenotypic methods. ESCr-EK isolates were subject to whole genome sequencing. We calculated prevalence and adjusted prevalence ratios (aPR) using binomial regression.
\end{abstract}

Results: Of 747 patients, 45 (6.0\%) were colonised with ESCr-EK, none with VRE. The ESCr-EK isolates in 41 patients were multidrug resistant; no isolates were non-suceptible to meropenem. Prevalence of ESCr-EK was higher among travellers to Asia (aPR $=6.6 ; 95 \% \mathrm{Cl} 3.6-12 ; p<0.001$ ). No statistical significant difference in carriage was observed between departments, age or any other factors in the univariable analyses.

Conclusions: The observed prevalence of ESCr-EK colonisation upon admission was in the same range but lower than that reported in similar studies from Europe. Travel to Asia was a strong predictor for colonisation of ESCr-EK to be considered when administering empirical antimicrobial treatment. As less than one third of colonised patients had travelled to Asia, and no other factors investigated were found to be strongly associated with carriage, these findings underscore that healthcare personnel must apply standard infection control precautions for all patients.

Keywords: Prevalence, Beta-lactam resistance, Hospitals, university, Cross-sectional, Studies, Enterobacteriaceae, Epidemiology, Drug resistance, multiple

\footnotetext{
* Correspondence: laura_espenhain@hotmail.com

'Department of Antibiotic Resistance and Infection Prevention, Norwegian

Institute of Public Health, PO Box 222 Skøyen, 0213 Oslo, NO, Norway

2European Programme for Intervention Epidemiology Training (EPIET),

European Centre for Disease Prevention and Control, (ECDC), Stockholm,

Sweden

Full list of author information is available at the end of the article
}

(C) The Author(s). 2018 Open Access This article is distributed under the terms of the Creative Commons Attribution 4.0 International License (http://creativecommons.org/licenses/by/4.0/), which permits unrestricted use, distribution, and reproduction in any medium, provided you give appropriate credit to the original author(s) and the source, provide a link to the Creative Commons license, and indicate if changes were made. The Creative Commons Public Domain Dedication waiver (http://creativecommons.org/publicdomain/zero/1.0/) applies to the data made available in this article, unless otherwise stated. 


\section{Background}

Escherichia coli and other Enterobacteriaceae frequently cause nosocomial and community acquired infections [1] and the burden due to infection with Enterobacteriaceae resistant to extended-spectrum cephalosporins (ESC) is increasing worldwide [2]. Infections are often preceded by colonisation of the gut with these bacteria. Among Enterobacteriaceae, resistance to ESC is often caused by production of extended-spectrum beta-lactamases (ESBLs) or plasmid mediated AmpC (pAmpC) [2]. In a hospital setting, carriage of Enterobacteriaceae resistant to ESC (ESCr-E) is not only a concern for the individual patient, but also for the surrounding patients as these bacteria can spread via the faecal-oral route between patients within the hospital [3]. In addition, plasmids conferring ESC resistance can be transferred between different bacterial strains and species. Outbreaks of ESBL-producing E. coli and Klebsiella spp. have been reported in healthcare institutions in Norway [4-6]. Yet another concern is that ESC-resistance often occurs along with co-resistance to important non-beta-lactam antibiotic classes [7], significantly limiting treatment options for these patients.

Enterobacteriaceae are part of the normal gut flora and pose a complex challenge as eradication is problematic [8]. If introduced within a hospital, infection prevention and control rely on strict compliance to standard precautions $[3,9]$. The Norwegian Institute of Public Health (NIPH) recommends isolation of patients in general hospital wards if they carry carbapenemase producing Enterobacteriaceae or vancomycin resistant enterococci. Patients who carry ESC resistant Enterobacteriaceae should be isolated if admitted to departments with particularly vulnerable patients (neonatal, cancer or intensive care units) [10]. Even though the Nordic countries have a low prevalence of antimicrobial resistance among Enterobacteriaceae, resistance to ESC in E. coli and Klebsiella ssp in urine and blood specimens is increasing [11]. In order to better target interventions to prevent spread between patients and to guide empirical antibiotic treatment we aimed to estimate the prevalence of faecal carriage of ESC resistant E. coli and $K$. pneumoniae (ESCr-EK) and vancomycin resistant enterococci (VRE) in patients upon hospital admission, and to identify factors associated with carriage. Additionally we aimed to explore the diversity of ESCr-EK and determine the genetic background for resistance to ESC.

\section{Methods}

\section{Design, setting and study population}

The cross-sectional study was carried out at Akershus University Hospital, a secondary care facility located in a mixed urban and rural area east of the Norwegian capital Oslo. The catchment area covers around $10 \%$ (500,000 persons) of the Norwegian population. Trained nurses recruited patients upon admission. In the adult's emergency department, recruitment took place on selected weekdays between October 2014 and July 2015, all patients presenting between 8 am and 11 am were invited to participate. In the children's emergency department, all patients/parents admitted during selected weeks in the period between January 2015and July 2016 were invited to participate. From October 2015 to December 2016 recruitment was expanded to three surgical units (thoracic, urology, and orthopaedic) where all patients presenting for pre-surgical examination prior to elective surgery were invited to participate.

Patients with suspected acute coronary disease, in need of immediate intensive care, and adult patients unable to give informed consent were not recruited.

\section{Data collection}

Consenting patients or parents of patients below 16 years of age completed a questionnaire with 26 questions, including demographics and possible risk factors for colonisation with ESCr-EK such as travel outside of the Nordic countries, antibiotic consumption, health care and medical devices within the 12 months prior to recruitment. Only information about risk factors prior to admission was collected. One rectal swab was collected at admission or maximum $24 \mathrm{~h}$ after admission.

\section{Microbiology}

Rectal swabs were collected and transported in liquid medium (eSwab, Copan, Italy). The samples were stored at $4{ }^{\circ} \mathrm{C}$ for maximum $14 \mathrm{~h}$, with subsequent plating on blood agar for growth control, and on two selective lactose agar plates with the addition of either $1 \mathrm{mg} / \mathrm{ml}$ cefotaxime or $1 \mathrm{mg} / \mathrm{ml}$ ceftazidime for detection of ESCr-EK, produced in-house. For the detection of vancomycin resistant Enterococci, we used Brilliance VRE plates (Oxoid, ThermoFisher Scientific, MA, USA). Morphologically distinct colonies from each selective plate were subcultured, and identified by MALDI-TOF mass spectrometry (MALDI-TOF, Bruker Daltonics, Bremen, Germany). Isolates of E. coli or Klebsiella spp. were frozen at $-80{ }^{\circ} \mathrm{C}$ for later analysis. We did susceptibility disk testing and interpretation of clinical breakpoints according to EUCAST methodology (http:// www.eucast.org/fileadmin/src/media/PDFs/EUCAST_files/ Breakpoint_tables/Breakpoint_table_v_4.0.xls, 2017), and divided the antibacterial agents into six classes: Penicillins (Ampicillin), cephalosporins and penicillins in combination with betalactam-inhibitors (cefoxitin, cefotaxime, ceftazidime and piperacillin-tazobactam), carbapenems (meropenem), (fluoro)quinolones (ciprofloxacine and nalidixin), aminoglycosides (gentamicin), and folate-pathway inhibitors (trimethoprim-sulphamethoxazole). We defined multidrug resistance as non-susceptibility (i.e. resistant or intermediate susceptible) to three or more of the above antibiotic classes. 
From each patient positive for ESCr-EK, one isolate was selected for whole genome sequencing (WGS). If the patient carried both ESC resistant E. coli and Klebsiella spp., one isolate per species was included for WGS. Furthermore, if a patient carried several morphologically different strains of the same species, and if the strains had different antimicrobial susceptibility patterns (indicative of polyclonal carriage), one representative isolate for each pattern was included for WGS. WGS was done with Illumina technology (Illumina, San-Diego, USA), and data were analysed on the publicly available platform at the Center for Genomic Epidemiology [12]. Default threshold settings were used for MLST and ResFinder [13, 14].

\section{Statistical analysis}

We expressed the prevalence of ESCr-EK upon admission as the number of patients positive for ESCr-EK per 100 patients enrolled in the study. For the descriptive analysis, and to evaluate independent factors associated with colonisation we performed uni- and multivariable binominal regression analyses. We grouped countries into five regions (Europe, Asia, Americas, Africa and Australia). The category "Asia" included countries in the Middle-East as well as Central and East Asia (listed in Additional file 1). In the multivariable analyses variables with a $p$-value of $<0.2$ were included in the model one by one starting with the factor with the highest prevalence ratio. Model fit was evaluated using the likelihood-ratio test. $P$-values $<0.05$ were considered significant. Health care outside of the Nordic countries, included in the national screening guidelines, was included in the final model irrespective of level of significance.

\section{Human subject protection}

Informed consent was obtained from each patient or parents of patients below 16 years of age. The Regional Committees for Medical and Health Research Ethics (REC 2012/ 2234) and the hospital's privacy protection officer approved the study protocol. The study was funded by the NIPH.

\section{Results}

\section{Participation}

In total, 747 rectal swabs accompanied by a completed questionnaire were collected from patients upon admission. Among the participants, 391 (52\%) participants were male, ranging from $43 \%$ in the orthopaedic unit to $62 \%$ in the urology unit. Twenty per cent were below three years of age and $39 \%$ were 65 years or older. An estimate of $84 \%$ of patients invited to participate accepted.

\section{Microbiology}

Patients

Of the 747 rectal swabs, 45 (6.0\%) contained one or more isolate resistant to cefotaxime and/or ceftazidime. E. coli was found in 43 of the 45 samples and K. pneumoniae in five, i.e. both ESC resistant E. coli and $K$. pneumoniae were identified in three samples. Co-resistance to fluoroquinolones, piperacillin-tazobactam and gentamicin was observed in ESCr-EK isolates from 39, 11 and 10 patients, respectively. The ESCr-EK isolates in 41 patients were multidrug resistant. No patients carried isolates non-susceptible to meropenem. Carriage of vancomycin resistant enterococci was not detected in any of the participants.

\section{Isolates}

In total, $57 \mathrm{ESCr}$-EK isolates from the $45 \mathrm{ESCr}$-EK carriers were subject to WGS. Among these, 51 isolates from 42 patients harboured one or more ESBL genes of the bla $a_{\text {CTX-M groups }}$ (5.6\% of all patients) and five isolates from four patients harboured pAmpC genes $\left(b l a_{\mathrm{DHA}-1}\right.$ or $\left.b l a_{\mathrm{CMY}-42}\right)$ (Table 1). In one patient both bla $a_{\mathrm{CTX}-\mathrm{M}}$ and $b l a_{\mathrm{DHA}-1}$ were identified in different isolates. One isolate harboured both $b l a_{\mathrm{CTX}-\mathrm{M}-15}$ and $b l a_{\mathrm{CMY}-42}$. In two isolates, one E. coli and one $K$. pneumoniae, ResFinder v3.0 (accessed July 27th 2018) did not detect any resistance mechanism to explain ESC resistance.

Among the 57 isolates that were subject to WGS, 25 different MLSTs were identified in E.coli and two in $K$. pneumoniae. One fourth of the E. coli [14] belonged to ST131 (Table 2). Three patients carried several E. coli strains with different MLSTs. Nine MLSTs (34, 83, 93, $99,349,398,410,485$, and 617) were found only in patients who had solely travelled to Asia.

\section{Epidemiology}

In the univariable analyses (Table 3), we observed a significantly higher prevalence of ESCr-EK in patients who had travelled to Asia (33\%) compared with those who had not travelled to Asia (4.5\%), in patients who had travelled outside of the Nordic countries (11\%) compared to patients who had not (3.9\%), and in patients

Table 1 Genotypes of ESCr-EK isolates ( $n=52$ E.coli and $5 \mathrm{~K}$. pneumoniae) from 45 ESCr-EK carriers

\begin{tabular}{|c|c|c|c|}
\hline Enzyme & gene identified & E. coli & K. pneumoniae \\
\hline \multirow[t]{8}{*}{$\overline{E S B L}$} & bla $a_{\mathrm{CTX}-\mathrm{M}-15}$ & 28 & 1 \\
\hline & bla $a_{\mathrm{CTX}-\mathrm{M}-55}$ & 6 & \\
\hline & $b l a_{C T X-M-27}$ & 6 & \\
\hline & $b / a_{O \times A-1}{ }^{a}$ & 8 & \\
\hline & $b l a_{C T X-M-14(+b)}$ & 5 & \\
\hline & $b l a_{C T X-M-1}$ & 4 & \\
\hline & $b l a_{C T X-M-3}$ & 1 & 1 \\
\hline & $b / a_{\text {TEM- }-33}$ & 1 & \\
\hline \multirow[t]{2}{*}{ pAmpC } & $b / a_{\mathrm{DHA}-1}$ & 2 & 2 \\
\hline & $b / a_{\mathrm{CMY}-42}$ & 1 & \\
\hline Uncertain & None detected & 1 & 1 \\
\hline
\end{tabular}

${ }^{a}$ bla $a_{-\mathrm{OXA}-1}$ always occurring in isolates also harbouring $b / a_{\mathrm{CTX}-\mathrm{M}-15}$ 
Table 2 Distribution of MLST types ESCr-EK isolates ( $n=52$ E.coli and $5 \mathrm{~K}$. pneumoniae) from 45 ESCr-EK carriers

\begin{tabular}{|c|c|c|}
\hline MLST type & E. coli & K. pneumoniae \\
\hline 131 & 14 & \\
\hline 38 & 5 & \\
\hline 405 & 4 & \\
\hline 10 & 2 & \\
\hline 34 & 2 & \\
\hline 69 & 2 & \\
\hline 83 & 2 & \\
\hline 357 & 2 & \\
\hline 648 & 2 & \\
\hline Unknown MLST ${ }^{a}$ & 2 & 3 \\
\hline Other MLST types ${ }^{\mathrm{b}}$ & 15 & 2 \\
\hline Total & 52 & 5 \\
\hline
\end{tabular}

${ }^{\mathrm{a} M L S T}$ not resolved

bE. coli: MLST48, 73, 93, 99, 167, 394, 398, 410, 485, 617, 1163, 1193, 1611, 1722, 3036. K. pneumoniae: MLST37, 1427

who had received health care outside the Nordic countries (14\%) compared to those who had not (5.6\%). A higher, but non-significant, prevalence was observed in patients born outside Norway (11\%) compared to patients born in Norway (5.9\%), and in those who had travelled to Africa (18\%) compared to those who had not (5.8\%). No statistical significant difference in carriage was observed between departments of recruitment or age in years or age group or any other factors in the univariable analyses (Table 3 ).

In the multivariable analyses, travel to Asia was the only factor associated with carriage. Health care outside the Nordic countries was also included in the reported model as it is part of the national screening recommendations. The binominal regression analysis showed that travellers to Asia had a significantly higher prevalence of ESCr-EK carriage compared to those who had not travelled (PR 6.1; 95\% CI 3.4-11; $p<0.001$ ) (Table 4). Travel to Asia was primarily to Thailand $(n=17)$, Turkey $(n=17)$, and Pakistan $(n=6)$.

\section{Discussion}

In this study, including 747 patients from two emergency departments and three surgical units, we found no E.coli or Klebsiella spp. resistant to carbapenems, and no vancomycin resistant enterococci. The prevalence of colonisation of ESCr-EK upon admission was $6.0 \%$. A major predictor for colonisation was travel to Asia. However, the majority of colonised patients had not been to Asia 12 months prior to admission.

The observed prevalence of ESCr-EK (6.0\%) and ESBL-EK colonisation (5.6\%) at admission was in the same range but lower than reported in similar studies from Germany (7.5-9.5\%) [15], the Netherlands $(8.2 \%)$ [16], and Israel (10.8\%) [17] and slightly higher than previously reported in healthy individuals in Sweden (4.7\%) [18] and Norway (4.9\%) [19]. Varying methods and definitions challenge direct comparison between studies and this may, at least in part, explain these differences between countries. Other explanations may be differences in age, ethnicity, co-morbidity, previous hospital admissions or a true lower prevalence upon admission in the Norwegian population compared to the prevalence in the Netherlands, Germany or Israel. To our knowledge, there has been no previous Norwegian studies of ESBL prevalence in patients at admittance to hospital.

Self-reported use of antibiotics has been found to be a risk factor for ESC-E colonisation at admission in Germany [15]. We could not confirm this finding, nor could studies from the Netherlands [16] and Sweden [18]. Differing antibiotic-prescription policies may be one explanation for this. It was not possible to perform stratified analyses on antibiotics class as one third of participants who had used antibiotics 12 months prior to the admission did not indicate which class.

Consistent with other studies, visiting Asia within 12 months prior to faecal sampling, was associated with ESCr-EK colonisation (PR 6.8, $p<0.001$ ). Prevalence ranging from 13 to $45 \%$ has been found in Swedish [18, 20] and Danish [21] travellers to Asia. In accordance with our study, none of these studies found carbapenem-resistant Enterobacteriaceae. However, the methods we used were not optimised to detect carbapenemases like OXA-48, which do not also confer resistance to cefotaxime or ceftazidime. The higher prevalence in patients who had travelled to Asia is also consistent with results from prevalence studies from Asia (Pakistan [22], Thailand [23-25], Turkey [26]) on different populations and types of specimens, where a prevalence of up to $40-77 \%$ has been reported even in healthy adults and food products.

This study covers a convenience sample of a population utilizing the hospital. We recruited patients from two emergency departments and three elective surgery units, and a wide range of ages from infancy to elderly patients. It was not practically feasible to recruit critically ill patients admitted to intensive care units in the hospital. Hence, we do not describe the prevalence of ESCr-EK in units with patients at the higher risk for a severe outcome if colonised with resistant bacteria. The prevalence tended to be higher amongst patients who reported having received health care outside of the Nordic countries. However, there were not enough patients in this category to give sufficient power to quantify a difference. The data collected was not detailed to a level allowing for stratification on type of health care (hospital admission, dental care, primary health care, etc.). 
Table 3 Number of patients, carriers, percent carriers, crude prevalence ratio (PR) and $p$-values from the univariable binominal regressions by risk factors for ESCr-EK carriage up to 12 months prior to admission $(n=747)$

\begin{tabular}{|c|c|c|c|c|c|c|}
\hline & & & Carriage of $\mathrm{ESCr}$ & & & \\
\hline & & $\begin{array}{l}\text { Total no. of } \\
\text { patients }\end{array}$ & No. of patients & $\overline{(\%)}$ & $\begin{array}{l}\text { Prevalence } \\
\text { ratio (PR) }\end{array}$ & $p$-value \\
\hline & & 747 & 45 & $(6.0 \%)$ & & \\
\hline Demography & Female & 356 & 16 & $(4.5 \%)$ & & \\
\hline & Male & 391 & 29 & $(7.4 \%)$ & 1.65 & 0.10 \\
\hline & 0-17 year & 222 & 11 & $(4.9 \%)$ & & \\
\hline & $18-64$ years & 233 & 18 & $(7.7 \%)$ & 1.81 & 0.10 \\
\hline & $65+$ years & 292 & 16 & $(5.5 \%)$ & 1.17 & 0.67 \\
\hline & Born in Norway & 675 & 40 & $(5.9 \%)$ & & \\
\hline & Not born in Norway & 45 & 5 & $(11.1 \%)$ & 1.88 & 0.16 \\
\hline & Parents born in Norway & 593 & 35 & $(5.9 \%)$ & & \\
\hline & One or both parents not born in Norway & 114 & 8 & $(7.0 \%)$ & 1.19 & 0.65 \\
\hline Department of inclusion & Emergency department (adult) & 122 & 11 & $(9.0 \%)$ & & \\
\hline & Emergency department (children) & 222 & 11 & $(4.9 \%)$ & 0.56 & 0.14 \\
\hline & Thoracic unit & 194 & 11 & $(5.7 \%)$ & 0.63 & 0.26 \\
\hline & Orthopaedic unit & 141 & 7 & $(5.0 \%)$ & 0.56 & 0.20 \\
\hline & Urology unit & 68 & 5 & $(7.3 \%)$ & 0.81 & 0.69 \\
\hline Exposures 12 months prior & No travel outside of the Nordic countries ${ }^{a}$ & 512 & 20 & $(3.9 \%)$ & 0.37 & 0.00 \\
\hline & Travel to Europe (vs. no travel to Europe) & 175 & 11 & $(6.3 \%)$ & 1.1 & 0.87 \\
\hline & Travel to America (vs. no travel to America) & 22 & 1 & $(4.6 \%)$ & 0.75 & 0.77 \\
\hline & Travel to Africa (vs. no travel to Africa) & 11 & 2 & $(18.2 \%)$ & 3.11 & 0.08 \\
\hline & Travel to Asia (vs. no travel to Asia) & 51 & 15 & $(29.4 \%)$ & 6.82 & 0.00 \\
\hline & No health care (vs. health care) & 499 & 32 & $(6.4 \%)$ & 0.82 & 0.55 \\
\hline & Admitted to a Norwegian hospital $^{b}$ & 224 & 11 & $(4.9 \%)$ & 0.81 & 0.55 \\
\hline & $\begin{array}{l}\text { Medical examination or treatment outside } \\
\text { the Nordic countries }{ }^{c}\end{array}$ & 28 & 4 & $(14.3 \%)$ & 2.54 & 0.05 \\
\hline & Work in health care (vs. other type of job) & 38 & 3 & $(7.9 \%)$ & 0.95 & 0.94 \\
\hline & Work in farming & 11 & 0 & $(0.0 \%)$ & - & - \\
\hline & Other type of job & 193 & 16 & $(8.3 \%)$ & & \\
\hline & No contact to animals at work & 188 & 17 & $(9.0 \%)$ & & \\
\hline & Contact to animals at work & 22 & 1 & $(4.5 \%)$ & 0.50 & 0.49 \\
\hline & No permanent urinary catheter & 668 & 40 & $(6.0 \%)$ & & \\
\hline & Permanent urinary catheter & 10 & 0 & $(0.0 \%)$ & - & - \\
\hline & $\begin{array}{l}\text { No medical devices put through skin/mucosa } \\
\text { before admission }\end{array}$ & 639 & 39 & $(6.1 \%)$ & & \\
\hline & Medical devices put through skin/mucosa & 46 & 2 & $(4.3 \%)$ & 0.71 & 0.63 \\
\hline & No wound or skin infections & 616 & 34 & $(5.5 \%)$ & & \\
\hline & Wound or skin infections & 76 & 6 & $(7.9 \%)$ & 1.43 & 0.40 \\
\hline & No antibiotic consumption & 515 & 28 & $(5.4 \%)$ & & \\
\hline & Antibiotic consumption & 232 & 17 & $(7.3 \%)$ & 1.35 & 0.31 \\
\hline
\end{tabular}

\footnotetext{
a(vs. travel outside of the Nordic countries)

b (vs. not admitted to a Norwegian hospital)

${ }^{c}$ (vs. no medical examination or treatment outside the Nordic countries)
} 
Table 4 Number of patients, carriers and adjusted prevalence ratio (aPR), 95\% Cl and p-values from the multivariable binominal regression model by risk factors for ESCr-EK carriage up to 12 months prior to admission $(n=741)$

\begin{tabular}{|c|c|c|c|c|c|}
\hline & Total & Number of ESCr-EK carriers & $\mathrm{aPR}$ & $95 \% \mathrm{Cl}$ & $p$-value \\
\hline Number of patients & 741 & 44 & & & \\
\hline Travel to Asia the past 12 months & 50 & 14 & 6.1 & $(3.43-11)$ & $<0.001$ \\
\hline Medical examination or treatment outside the Nordic countries & 28 & 4 & 1.7 & $(0.74-3.9)$ & 0.212 \\
\hline
\end{tabular}

aPR Adjusted prevalence ratio

The genetic mechanisms behind ESC resistance was mainly ESBL production due to $b l a_{\text {СTX-M. }}$. Only two ESCr-EK could not be explained by ESBL or pAmpC production. We found a large diversity of MLSTs in the 45 patients carrying ESCr-EK, making comparison with previous findings challenging. Sixteen different MLSTs were found in patients who had travelled to Asia, nine of these were found in travellers who had only been in Asia. However, many of these MLSTs represent well-known clones that have been found in various sources in several countries, including in Norway [7]. The dominant ESBL allele was $b l a_{\text {CTX-M-15 }}$ which has emerged and disseminated worldwide [27] and has previously been found in travellers returning from all over the world [20], and in environmental samples from our area [7]. The ESBL allele $b l a_{\mathrm{CTX}-\mathrm{M}-55}$ has been found to be one of the most commonly encountered ESBL-encoding genes in Enterobacteriaceae from food animals in China and has also been found in food animals in Europe together with other CTX-M types [28, 29] suggesting the potential importance of animal-to-human spread via contaminated food [20].

In our study, travel to Asia was primarily to Thailand and Pakistan. We expect that travel to other high endemic areas in Asia like India, or to Africa as found in other studies $[18,20]$, might also be a risk factor for colonisation as also indicated in our study. All the ESCr-EK isolates from patients who had travelled to Asia were multidrug resistant.

Urinary tract infections and bacteraemia are often preceded by colonisation of the gut $[17,30]$. As more than one third of patients who had travelled to Asia within the last 12 months carried ESCr-EK, we would recommend that these patients receive empiric antimicrobial treatment effective against ESCr-EK if they present with signs of serious infections of possible Gram-negative origin. According to findings in several studies, and repeated here, ESCr-EK are often multidrug resistant, and carbapenems is probably the safest choice for empirical treatment [31]. Treatment should always be adjusted and, if possible, de-escalated as soon as culture results are available [32, 33].

Certain ESCr-EK phylogroups or subclones have been linked to increased potential to cause severe infections because of higher virulence [18]. More knowledge of the more virulent phylogroups or subclones and risk factors for colonisation would allow targeted screening/treatment guidelines. As of today, the national guidelines for ESCr-EK infection control and prevention do not differentiate between MLSTs.

\section{Conclusion}

The prevalence of colonisation of ESCr-EK upon admission was $6.0 \%$, none of the isolates identified from the patients produced carbapenemases and we did not identify any VRE, supporting other surveillance studies which indicate that carbapenemase-producing E. coli and Klebsiella and VRE are still very uncommon in the Norwegian population [11, 19]. Travel to Asia was a strong predictor for colonisation of ESCr-EK to be considered when administering empiric antimicrobial treatment. Less than one third of the colonised patients had travelled to Asia, and no other factors investigated were found to be strongly associated with carriage. For infection prevention and control, these findings underscore that health care personnel cannot rely solely on risk assessment and screening results but must apply standard infection control precautions for all patients.

\section{Additional file}

Additional file 1: Countries of Asia and number of participants who have travelled there $(n=747)$. (DOCX $29 \mathrm{~kb})$

\section{Acknowledgements}

Not applicable

\section{Funding}

The study was funded by the NIPH.

\section{Availability of data and materials}

The data from questionnaires that support the findings of this study are available from Akershus University Hospital but restrictions apply to the availability of these data, which were used under license for the current study, and so are not publicly available. Data are however available from the authors upon reasonable request and with permission of the Regional Ethics Committee of South-Eastern Norway.

\section{Authors' contributions}

SBJ and MSt design the study, MML, BN and SBJ took part in the recruitment of participants and collection of questionnaires and swabs. $\mathrm{SHH}$ analysed the samples and interpreted the results. LE combined, analysed and interpreted the laboratory and epidemiological data. SBJ double checked and verified any discrepancy in the data. MSu analysed the wgs output. LE drafted the 
manuscript and SBJ, MSu and MSt revised it critically. TML and BN revised the advanced manuscript. All authors read and approved the final manuscript.

\section{Ethics approval and consent to participate}

The Regional Committees for Medical and Health Research Ethics (REC 2012/ 2234) and the privacy protection officer at Akershus University hospital approved the study protocol. Informed consent was obtained from each patient or parents of patients below 16 years of age.

\section{Consent for publication}

Not applicable

\section{Competing interests}

The authors LE, SBJ, TML, MSU, SHH and MSt declare that they have no competing interests. BN has received funding from MSD as national lead invesgitator in Phamacokinetics Phase 1 study MK 1986 (Tedizolid) and 7655 (combination of imipenem, cilastatin and relebactam) for children outside of the submitted work.

\section{Publisher's Note}

Springer Nature remains neutral with regard to jurisdictional claims in published maps and institutional affiliations.

\begin{abstract}
Author details
'Department of Antibiotic Resistance and Infection Prevention, Norwegian Institute of Public Health, PO Box 222 Skøyen, 0213 Oslo, NO, Norway. ${ }^{2}$ European Programme for Intervention Epidemiology Training (EPIET), European Centre for Disease Prevention and Control, (ECDC), Stockholm, Sweden. ${ }^{3}$ Department of Clinical Microbiology and Infection Control, Akershus University Hospital, Lørenskog, Norway. ${ }^{4}$ Institute for Clinical Medicine, Campus Ahus, University of Oslo, Nordbyhagen, Norway. ${ }^{5}$ Department of Paediatrics and Adolescents Medicine, Akershus University Hospital, Lørenskog, Norway. ${ }^{6}$ Department of Molecular Biology, Norwegian Institute of Public Health, Oslo, Norway. ${ }^{7}$ Section for Food Safety and Emerging Health Threats, Norwegian Veterinary Institute, Nordbyhagen, Norway.
\end{abstract}

Received: 23 August 2018 Accepted: 30 October 2018 Published online: 29 November 2018

\section{References}

1. World Health Organisation. Antimicrobial resistance: global report on surveillance. World Health Organization. Geneva: WHO, 2014. ISBN 978924 1564748

2. de Kraker ME, Jarlier V, Monen JC, Heuer OE, van de Sande N, Grundmann $\mathrm{H}$. The changing epidemiology of bacteraemias in Europe: trends from the European antimicrobial resistance surveillance system. Clin Microbiol Infect. 2013;19(9):860-8.

3. Tacconelli E, Cataldo MA, Dancer SJ, De Angelis G, Falcone M, Frank U, et al. ESCMID guidelines for the management of the infection control measures to reduce transmission of multidrug-resistant gram-negative bacteria in hospitalized patients. Clin Microbiol Infect. 2014;20(Suppl 1):1-55.

4. Naseer U, Natas OB, Haldorsen BC, Bue B, Grundt H, Walsh TR, et al. Nosocomial outbreak of CTX-M-15-producing E. coli in Norway. APMIS. 2007;115(2):120-6.

5. Jorgensen SB, Bojer MS, Boll EJ, Martin Y, Helmersen K, Skogstad M, et al. Heat-resistant, extended-spectrum beta-lactamase-producing Klebsiella pneumoniae in endoscope-mediated outbreak. J Hosp Infect. 2016;93(1):57-62.

6. Tofteland S, Naseer U, Lislevand JH, Sundsfjord A, Samuelsen O. A long-term low-frequency hospital outbreak of KPC-producing Klebsiella pneumoniae involving Intergenus plasmid diffusion and a persisting environmental reservoir. PLoS One. 2013;8(3):e59015.

7. Jorgensen SB, Soraas AV, Arnesen LS, Leegaard TM, Sundsfjord A, Jenum PA A comparison of extended spectrum beta-lactamase producing Escherichia coli from clinical, recreational water and wastewater samples associated in time and location. PLoS one [internet]. 2017 PMC5645111]; 12(10):[e0186576 p.]. Available from: https://doi.org/10.1371/journal.pone.0186576.

8. Biehl LM, Schmidt-Hieber M, Liss B, Cornely OA, Vehreschild MJ. Colonization and infection with extended spectrum beta-lactamase producing Enterobacteriaceae in high-risk patients - review of the literature from a clinical perspective. Crit Rev Microbiol. 2016;42(1):1-16.
9. European Center for Disease Control and Prevention. Systematic review of the effectiveness of infection control measures to prevent the transmission of extended-spectrum beta-lactamase-producing Enterobacteriaceae through cross-border transfer of patients. Stockholm: European Centre for Disease Prevention and Control; 2014.

10. Folkehelseinstitutttet. ESBL-holdige gramnegative stavbakterier smitteverntiltak i helseinstitusjoner2015.

11. NORM/NORM-VET2016. Usage of Antimicrobial Agents and Occurrence of Antimicrobial Resistance in Norway. Tromsø/Oslo: NORM/NORM-VET, 2017 1890-9965.

12. Center for Genomic Epidemiology. [cited 201827 July]. Available from: www.genomicepidemiology.org.

13. Zankari E, Hasman H, Cosentino S, Vestergaard M, Rasmussen S, Lund O, et al. Identification of acquired antimicrobial resistance genes. J Antimicrob Chemother. 2012;67(11):2640-4.

14. Larsen MV, Cosentino S, Rasmussen S, Friis C, Hasman H, Marvig RL, et al. Multilocus sequence typing of total-genome-sequenced bacteria. J Clin Microbiol. 2012;50(4):1355-61.

15. Hamprecht A, Rohde AM, Behnke M, Feihl S, Gastmeier P, Gebhardt F, et al. Colonization with third-generation cephalosporin-resistant Enterobacteriaceae on hospital admission: prevalence and risk factors. J Antimicrob Chemother. 2016;71(10):2957-63.

16. Platteel TN, Leverstein-van Hall MA, Cohen Stuart JW, Thijsen SF, Mascini EM, van Hees BC, et al. Predicting carriage with extended-spectrum betalactamase-producing bacteria at hospital admission: a cross-sectional study. Clin Microbiol Infect. 2015;21(2):141-6.

17. Ben-Ami R, Schwaber MJ, Navon-Venezia S, Schwartz D, Giladi M, Chmelnitsky l, et al. Influx of extended-spectrum beta-lactamase-producing enterobacteriaceae into the hospital. Clin Infect Dis. 2006;42(7):925-34.

18. Ny S, Lofmark S, Borjesson S, Englund S, Ringman M, Bergstrom J, et al. Community carriage of ESBL-producing Escherichia coli is associated with strains of low pathogenicity: a Swedish nationwide study. J Antimicrob Chemother. 2017;72(2):582-8.

19. Ulstad CR, Solheim M, Berg S, Lindbaek M, Dahle UR, Wester AL. Carriage of ESBL/AmpC-producing or ciprofloxacin non-susceptible Escherichia coli and Klebsiella spp in healthy people in Norway. Antimicrob Resist Infect Control. 2016;5:57.

20. Ostholm-Balkhed A, Tarnberg M, Nilsson M, Nilsson LE, Hanberger $H_{\text {, }}$ Hallgren A, et al. Travel-associated faecal colonization with ESBL-producing Enterobacteriaceae: incidence and risk factors. J Antimicrob Chemother [internet]. 2013;68(9):2144-53 pp.]. Available from: https://doi.org/10.1093/ jac/dkt167.

21. Lausch KR, Fuursted K, Larsen CS, Storgaard M. Colonisation with multiresistant Enterobacteriaceae in hospitalised Danish patients with a history of recent travel: a cross-sectional study. Travel Med Infect Dis. 2013;11(5):320-3.

22. Abrar S, Hussain S, Khan RA, UI Ain N, Haider H, Riaz S. Prevalence of extendedspectrum-beta-lactamase-producing Enterobacteriaceae: first systematic metaanalysis report from Pakistan. Antimicrob Resist Infect Control. 2018;7:26.

23. Boonyasiri A, Tangkoskul T, Seenama C, Saiyarin J, Tiengrim S, Thamlikitkul V. Prevalence of antibiotic resistant bacteria in healthy adults, foods, food animals, and the environment in selected areas in Thailand. Pathogens and global health. 2014;108(5):235-45.

24. Luvsansharav UO, Hirai I, Nakata A, Imura K, Yamauchi K, Niki M, et al. Prevalence of and risk factors associated with faecal carriage of CTX-M betalactamase-producing Enterobacteriaceae in rural Thai communities. J Antimicrob Chemother. 2012;67(7):1769-74.

25. Sasaki T, Hirai I, Niki M, Nakamura T, Komalamisra C, Maipanich W, et al. High prevalence of CTX-M beta-lactamase-producing Enterobacteriaceae in stool specimens obtained from healthy individuals in Thailand. J Antimicrob Chemother. 2010;65(4):666-8.

26. Tekiner $\mathrm{IH}$, Ozpinar $\mathrm{H}$. Occurrence and characteristics of extended spectrum beta-lactamases-producing Enterobacteriaceae from foods of animal origin. Braz J Microbiol. 2016;47(2):444-51.

27. Oteo J, Perez-Vazquez M, Campos J. Extended-spectrum [beta]-lactamase producing Escherichia coli: changing epidemiology and clinical impact. Curr Opin Infect Dis. 2010;23(4):320-6.

28. Ma J, Liu JH, Lv L, Zong Z, Sun Y, Zheng H, et al. Characterization of extended-spectrum beta-lactamase genes found among Escherichia coli isolates from duck and environmental samples obtained on a duck farm. Appl Environ Microbiol. 2012;78(10):3668-73. 
29. Snow LC, Warner RG, Cheney T, Wearing H, Stokes M, Harris K, et al. Risk factors associated with extended spectrum beta-lactamase Escherichia coli (CTX-M) on dairy farms in north West England and North Wales. Prev Vet Med. 2012:106(3-4):225-34.

30. Rodriguez-Bano J, Lopez-Cerero L, Navarro MD, Diaz de Alba P, Pascual A. Faecal carriage of extended-spectrum beta-lactamase-producing Escherichia coli: prevalence, risk factors and molecular epidemiology. J Antimicrob Chemother. 2008;62(5):1142-9.

31. Sheu CC, Lin SY, Chang YT, Lee CY, Chen YH, Hsueh PR. Management of infections caused by extended-spectrum beta-lactamase-producing Enterobacteriaceae: current evidence and future prospects. Expert Rev AntiInfect Ther. 2018;16(3):205-18.

32. Helsedirektoratet. Nasjonal faglig retningslinje for bruk av antibiotika i sykehus. In: Helsedirektoratet, editor. Oslo 2017.

33. Healthcare Infection Control Practices Advisory Committee. Antibiotic stewardship statement for antibiotic guidelines - recommendations of the healthcare infection control practices advisory (HICPAC) committee 2016.

Ready to submit your research? Choose BMC and benefit from:

- fast, convenient online submission

- thorough peer review by experienced researchers in your field

- rapid publication on acceptance

- support for research data, including large and complex data types

- gold Open Access which fosters wider collaboration and increased citations

- maximum visibility for your research: over $100 \mathrm{M}$ website views per year

At BMC, research is always in progress.

Learn more biomedcentral.com/submissions 